\title{
Determining Gibbs Energies of Adsorption from Corrosion Inhibition Efficiencies: Is it a reliable approach?
}

DOI:

10.1016/j.corsci.2019.04.040

Document Version

Accepted author manuscript

Link to publication record in Manchester Research Explorer

\section{Citation for published version (APA):}

Walczak, M., Morales Gil, P., \& Lindsay, R. (2019). Determining Gibbs Energies of Adsorption from Corrosion Inhibition Efficiencies: Is it a reliable approach? Corrosion Science. https://doi.org/10.1016/j.corsci.2019.04.040

\section{Published in:}

Corrosion Science

\section{Citing this paper}

Please note that where the full-text provided on Manchester Research Explorer is the Author Accepted Manuscript or Proof version this may differ from the final Published version. If citing, it is advised that you check and use the publisher's definitive version.

\section{General rights}

Copyright and moral rights for the publications made accessible in the Research Explorer are retained by the authors and/or other copyright owners and it is a condition of accessing publications that users recognise and abide by the legal requirements associated with these rights.

\section{Takedown policy}

If you believe that this document breaches copyright please refer to the University of Manchester's Takedown Procedures [http://man.ac.uk/04Y6Bo] or contact uml.scholarlycommunications@manchester.ac.uk providing relevant details, so we can investigate your claim.

\section{OPEN ACCESS}




\title{
Determining Gibbs Energies of Adsorption from Corrosion Inhibition Efficiencies: Is it a reliable approach?
}

\author{
Monika S. Walczak ${ }^{\mathrm{a}}$, Perla Morales-Gila ${ }^{\mathrm{a}, \mathrm{b}}$, Robert Lindsay ${ }^{\mathrm{a}, \mathrm{c}^{*}}$ \\ ${ }^{a}$ Corrosion and Protection Centre, School of Materials, The University of \\ Manchester, Sackville Street, Manchester M13 9PL, UK \\ ${ }^{b}$ Laboratorio de Caracterización de Materiales Sintéticos y Naturales, Instituto \\ Mexicano del Petróleo, Ciudad de México, México \\ cPhoton Science Institute, The University of Manchester, Manchester, M13 9PL, UK.
}

*Corresponding author: Robert Lindsay; Tel: +44 161306 4824; Fax: +44 161306 486; Email: robert.lindsay@manchester.ac.uk 


\begin{abstract}
The common practice of using inhibition efficiency $(\eta \%)$ to determine the standard Gibbs energy of adsorption ( $\Delta \mathrm{G}_{\text {ads }}^{\circ}$ ) for a corrosion inhibitor in acidic solution is evaluated. It is demonstrated that the typical assumption that $\eta \%$ is a good proxy for fractional surface coverage $(\theta)$ is not necessarily valid. Consequently, the accuracy of the $\Delta \mathrm{G}_{\text {ads }}^{\circ}$ value obtained from such data is doubtful. Moreover, it is argued that even a more direct measurement of $\theta$ may still not allow one to extract an accurate estimate of $\Delta \mathrm{G}_{\mathrm{ads}}^{0}$.
\end{abstract}

Keywords: Acid solutions (A); XPS (B); Acid inhibition (C); Interfaces (C) 


\section{Introduction}

Corrosion inhibition of carbon steel, as well as other metals and alloys, in acidic solution is typically achieved through the addition of organic reagents exhibiting appropriate head and tail groups to facilitate substrate binding [1,2]. It is generally reported that these species function by forming an adsorbed single-molecule thick layer on the metallic substrate, which interferes with the anodic and/or cathodic corrosion reactions [1-3]. Consequently, key parameters for corrosion inhibitor performance are perceived to include the fraction of the substrate covered and the strength of adsorption [1-3]. On this basis, many studies of corrosion inhibitors in acidic solutions determine values for surface coverage and adsorption thermodynamics; a search of the Web of Science database [4] reveals that there at least 200 pertinent publications since 2015. In this paper, we show that this effort is generally of the little worth, as the validity of the underlying assumptions are habitually not proven.

Initial evaluation of candidate reagents for corrosion inhibition usually involves some measurement (e.g. weight loss or electrochemical response) of the corrosion rate of the substrate as a function of reagent concentration in bulk solution. Subsequently, these data are normally employed to determine the inhibition efficiency $(\eta \%)$ of a candidate at each concentration:

$$
\eta \%=\frac{(U-I)}{U} \times 100
$$

$\mathrm{U}(\mathrm{I})$ is the uninhibited (inhibited) corrosion rate. $\eta \%$ can be considered to be a figure of merit for corrosion inhibition performance, with the best performing reagents displaying values above 95\% (see, for example, Ref. 5). Moreover, for corrosion 
inhibitors in acidic solution, $\eta \%$ is widely presumed to be directly proportional to fractional surface coverage $(\theta)$, where $\theta=1$ is a complete monolayer, i.e.

$\eta \%=100 \times \theta$.

Using this equation, it is straightforward to convert $\eta \%$ to $\theta$, and construct a graph of the resulting values as a function of corrosion inhibitor concentration in bulk solution ([CI]). In such a plot, $\theta$ is typically found to increase asymptotically with [CI], suggesting that surface coverage is governed by a dynamic equilibrium between the adsorbed corrosion inhibitor species and those in solution, and so can be described by an adsorption isotherm [6]. For example, the isotherm formulated by Langmuir [7] is often applied, which is defined as:

$\theta=\frac{\mathrm{K}_{\mathrm{eq}}[\mathrm{Cl}]}{\left(1+\mathrm{K}_{\mathrm{eq}}[\mathrm{Cl}]\right)}$

$\mathrm{K}_{\mathrm{eq}}$, which has units of $\mathrm{M}^{-1}$ assuming $[\mathrm{CI}]$ is given in molarity $(\mathrm{M})$, is the equilibrium constant for the corrosion inhibitor adsorption reaction, and can be determined by fitting $\theta$ versus $[\mathrm{CI}]$ data. Having obtained $\mathrm{K}_{\mathrm{eq}}$, one can easily calculate the standard Gibbs energy of adsorption ( $\Delta \mathrm{G}_{\text {ads }}^{\circ}$ ) of the corrosion inhibitor, using:

$\Delta \mathrm{G}_{\mathrm{ads}}^{\circ}=-\mathrm{RT} \ln \mathrm{K}_{\mathrm{eq}}$,

where $\mathrm{R}$ is the universal gas constant. (N.B. $\mathrm{K}_{\text {eq }}$ input into equation (4) should be unitless, being derived from the activity of the corrosion inhibitor in solution rather than its molar concentration. Here, we assume that the activity coefficient is equal to 1, so that the values of these two quantities are identical, i.e. can simply use the value of $\mathrm{K}_{\mathrm{eq}}$ resulting from equation (3) to calculate $\Delta \mathrm{G}_{\mathrm{ads}}^{\circ}$.) 
Evidently, the methodology outlined above to determine $K_{e q}$, and so $\Delta G_{\text {ads }}^{\circ}$, for a corrosion inhibitor from $\eta \%$ data is relatively simple to execute, and so is highly appealing. Accurate determination of these values, however, depends upon $\eta \% \propto \theta$, which is invariably not demonstrated experimentally. Moreover, even if a robust estimate of $\theta$ is achievable, a number of other requirements $[6,7,8]$ need to be satisfied for quantitatively rigorous application of the Langmuir isotherm, i.e.

(i) Maximum adsorbate coverage is a monolayer;

(ii) A dynamic equilibrium state has been achieved;

(iii) All adsorption sites are equivalent;

(iv) No adsorbate-adsorbate interactions perturb adsorption behavior.

One must demonstrate sufficient fulfilment of all four of these criteria to ensure that the data fitting has some substantive value in terms of outputting reliable thermodynamic quantities.

Here, we explicitly examine the validity of $\eta \% \propto \theta$ for an exemplar system, employing both $\eta \%$ data and surface coverage derived from X-ray photoelectron spectroscopy (XPS) to independently determine $\mathrm{K}_{\mathrm{eq}}$ and $\Delta \mathrm{G}_{\mathrm{ads}}^{\circ}$. Comparison of the results suggests that many of the previously published values of $\Delta \mathrm{G}_{\text {ads }}^{\circ}$ for corrosion inhibitor adsorption are questionable; if the underlying assumptions are incorrect, then the derived quantities are also likely to be incorrect. Furthermore, we discuss whether or not conditions (i) - (iv), listed above, are ever realized for the current systems of interest, i.e. surface adsorption of corrosion inhibitors in acidic conditions.

\section{Experimental Details}

$\eta \%$ values and surface coverages from XPS data, acquired from carbon steel immersed in $1 \mathrm{M} \mathrm{HCl}$ solution inhibited with a series of concentrations of 2- 
mercaptobenzimidazole (MBI), are employed for this work; we note that the maximum concentration of MBI ( $2 \mathrm{mM})$ used in this study was found to be just below this compound's solubility limit in $1 \mathrm{M} \mathrm{HCl}$, and so extended magnetic stirring ( $\geq 2$ h) at room temperature was undertaken to ensure complete dissolution. These data have been previously published elsewhere, including details of their collection $[9,10]$. Briefly, corrosion rate as a function of MBI concentration, was determined with linear polarisation resistance (LPR) measurements after $4 \mathrm{~h}$ of substrate immersion; this immersion period was selected as the open circuit potential (OCP) had become stable for all MBI concentrations studied (see Figure S1 in Supplementary Material), i.e. a steady state had been achieved. These corrosion rate values were then converted to $\eta \%$ using equation (1). XPS spectra were acquired from similarly immersed substrates using a Kratos Axis Ultra facility. A glove box/load lock system was used for sample introduction to avoid post immersion oxidation; details of this procedure are provided in [13]. Surface coverages were determined through the application of standard equations $[9,10]$, using data recorded at two photoelectron emission angles, namely $0^{\circ}$ (emission along the surface normal) and $50^{\circ}$.

\section{Results and Discussion}

Plots of both MBI $\eta \%$ (red markers) and XPS surface coverage (blue markers) as a function of MBI concentration in bulk solution ([MBI]) are shown in Figure 1 (a). The latter is derived from the sum of XPS signals from two different surface bound species, which we have previously attributed to the thiol and thione tautomeric forms of MBI (see inset) on the basis of the N 1s XPS profile [9,10]; it should be noted that there is substantive evidence from ab initio modelling that the surface thiol species is much more likely to be thiolate (cleavage of S-H bond) [11,12], and so henceforth we will refer to this species as thiolate. It is evident that although the two datasets display similar trends, they are not coincident. To facilitate further comparison, we have converted the $\eta \%$ and XPS surface coverage values to what a priori are 
presumed to be fractional surface coverages, and which will be referred to, henceforth, as $\theta_{\eta \%}$ and $\theta_{\mathrm{XPS}}^{\text {Sum }}$, respectively. For $\theta_{\eta \%}$, equation (2) was employed to affect the conversion from $\eta \%$. Concerning $\theta_{\text {XPS }}^{\text {Sum }}$, the highest surface coverage (i.e. $1.3 \times 10^{15}$ molecule.cm ${ }^{-2}$ at $2 \times 10^{-3} \mathrm{M} \mathrm{MBI}$ ) was equated to $\theta_{\mathrm{XPS}}^{\mathrm{Sum}}=1$. Justification for this assignment is derived from the substrate surface atom $(\mathrm{Fe})$ density being $\sim 1.9$ $\times 10^{15}$ atom. $\mathrm{cm}^{-2}$, i.e. each MBI molecule occupies $\sim 1.5 \mathrm{Fe}$ atoms, which appears to be a reasonable ratio for a saturated monolayer; the accuracy of this assumption can be debated, but does not invalidate the ensuing discussion.

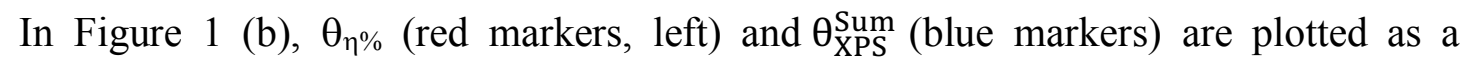
function of $[\mathrm{MBI}]$; the fractional XPS coverages of the thione ( $\theta_{\mathrm{XPS}}^{\text {Thione }}$, green markers) and thiolate ( $\theta_{\mathrm{XPS}}^{\text {Thiol }}$, purple markers) tautomeric components are also shown. Consistent with Figure 1 (a), these two supposed measures of fractional surface coverage are not equivalent, i.e. $\theta_{\eta \%} \not \equiv \theta_{\text {XPS }}^{\text {Sum }}$. For example, at an MBI concentration of $0.05 \times 10^{-3} \mathrm{M}, \theta_{\eta \%}$ is 0.94 , whereas $\theta_{\mathrm{XPS}}^{\mathrm{Sum}}$ is only 0.62 . As the surface coverage derived from XPS data provides a more direct estimate of this parameter, one may deduce that $\theta_{\eta} \%$ is not directly related to fractional surface coverage for this system. This conclusion directly contradicts the typical assumption for corrosion inhibition in acidic solution that $\eta \% \propto \theta$. Consequently, doubt is cast on the utility of $\theta_{\eta \%}$ for determination of thermodynamic quantities. A possible alternative interpretation of $\theta_{\eta \%}$, however, may still allow it to be used for this purpose, i.e. it is a measure of the fraction of active sites for corrosion that are quenched by the presence of the corrosion inhibitor. For example, at a surface fractional coverage of 0.62 (i.e. $\theta_{\mathrm{XPS}}^{\text {Sum }}=$ $0.62), 94 \%\left(\theta_{\eta \%}=0.94\right)$ of all the active corrosion sites are quenched, i.e. a complete monolayer $\left(\theta_{\mathrm{XPS}}^{\mathrm{Sum}}=1\right)$ is not required for MBI to achieve its maximum inhibition efficiency. If this definition of $\theta_{\eta} \%$ is valid, then one may still be able to reliably determine $\mathrm{K}_{\text {eq }}$ and $\Delta \mathrm{G}_{\text {ads }}^{\circ}$ for the active corrosion sites from such data. We test this hypothesis below. 


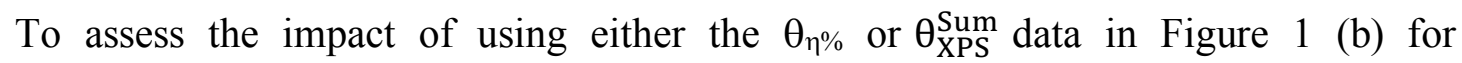
determination of $\mathrm{K}_{\mathrm{eq}}$, and thus $\Delta \mathrm{G}_{\mathrm{ads}}^{\circ}$, the equation for the Langmuir adsorption isotherm (equation (3)) has been employed to fit each dataset. Figure 2 (a) shows the

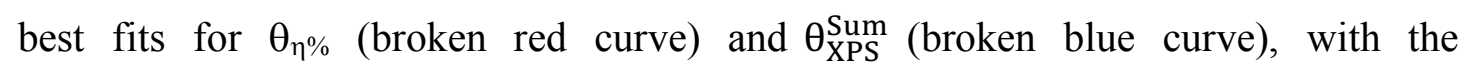
corresponding $\mathrm{K}_{\mathrm{eq}}$ and $\Delta \mathrm{G}_{\text {ads }}^{\circ}$ being shown in Figure 2 (b). Clearly, the two datasets result in significantly different values for these thermodynamic quantities, e.g. the difference between the two $\Delta \mathrm{G}_{\text {ads }}^{\circ}$ values is $5.5 \pm 0.9 \mathrm{kJmol}^{-1}$. One possible reason for this discrepancy is that only one of the adsorbed tautomeric forms, thione or thiolate, is quenching active corrosion sites. Hence, the adsorption isotherm for that particular component needs to be explicitly determined and compared to the $K_{\text {eq }}$ and $\Delta \mathrm{G}_{\text {ads }}^{\circ}$

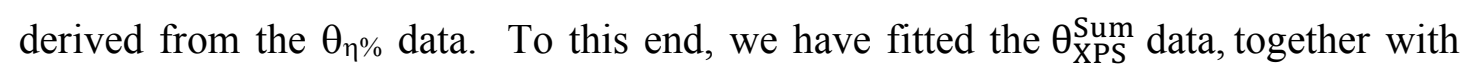
the fractional coverages of each tautomeric component $\left(\theta_{\mathrm{XPS}}^{\text {Thione }}\right.$ and $\left.\theta_{\mathrm{XPS}}^{\text {Thiol }}\right)$, with an expression involving the sum of two independent adsorption isotherms, each with a different $K_{\mathrm{eq}}$, i.e.

$\theta_{\mathrm{XPS}}^{\mathrm{Sum}}=\left(\mathrm{f} \times \frac{\left(\mathrm{K}_{\mathrm{eq}}^{\text {thione }}[\mathrm{MBI}]\right)}{\left(1+\mathrm{K}_{\mathrm{eq}}^{\text {thione }}[\mathrm{MBI}]\right)}\right)+\left((1-\mathrm{f}) \times \frac{\left(\mathrm{K}_{\mathrm{eq}}^{\text {thiol }}[\mathrm{MBI}]\right)}{\left(1+\mathrm{K}_{\mathrm{eq}}^{\text {thiol }}[\mathrm{MBI}]\right)}\right)$

The first term on the right hand side represents the Langmuir adsorption isotherm for the coverage of thione $\left(\theta_{\mathrm{XPS}}^{\text {Thione }}\right)$, with the second being for the coverage of thiolate $\left(\theta_{\mathrm{XPS}}^{\text {Thiol }}\right) . \mathrm{K}_{\mathrm{eq}}^{\text {thione }}$ and $\mathrm{K}_{\mathrm{eq}}^{\text {thiol }}$ are the equilibrium constants for the adsorption of the thione and thiolate tautomers, respectively. f defines the relative amounts of the two adsorbed tautomers $(0 \leq \mathrm{f} \leq 1)$, which is assumed to be independent of $[\mathrm{MBI}]$. We note that equation (5) differs from one stated in Ref. 6 for a multicomponent Langmuir adsorption isotherm, as a result of slightly different underlying assumptions. For the expression in Ref. 6, it is supposed that that all adsorption sites are equally available to all adsorbates. In contrast, we propose that active corrosion 
sites are only accessible to one of the two tautomers, i.e. only this specific tautomer is adsorbed at these sites.

Employing equation (5), and using $\mathrm{f}=0.6$ to be consistent with the asymptotic limit for $\theta_{\mathrm{XPS}}^{\text {Thione }}$, Figure 2 (c) displays the best fits for $\theta_{\mathrm{XPS}}^{\mathrm{Sum}}, \theta_{\mathrm{XPS}}^{\text {Thione }}$ and $\theta_{\mathrm{XPS}}^{\text {Thiol }}$. The corresponding thermodynamic quantities are listed in Figure 2 (d). Considering the associated errors, $\mathrm{K}_{\mathrm{eq}}$ and $\Delta \mathrm{G}_{\text {ads }}^{\circ}$ for the two tautomers are highly similar to each other as well as to the values determined from fitting of the $\theta_{\mathrm{XPS}}^{\text {Sum }}$ data with a single Langmuir isotherm (Figure 2 (b)). It should be noted that the slightly more exergonic $\Delta \mathrm{G}_{\text {ads }}^{\circ}$ for the thiolate tautomer is not incorrect, despite displaying consistently lower coverages than thione. This apparent anomaly is a product of the assumption underlying equation (5), i.e. one tautomer is adsorbed on one type of site on the surface (e.g. active corrosion sites), whilst the other adsorbs at a different type of site (e.g. non-active corrosion sites).

More importantly, none of the values listed in Figure 2 (d) are coincident with the $\mathrm{K}_{\mathrm{eq}}$ and $\Delta \mathrm{G}_{\text {ads }}^{\circ}$ values determined from the $\theta_{\eta \%}$ dataset; the smallest $\Delta \mathrm{G}_{\text {ads }}^{\circ}$ difference is 3.9 $\pm 2.6 \mathrm{kJmol}^{-1}$. As XPS is a more direct measure of surface coverage than $\theta_{\eta} \%$ data, one should therefore be highly wary about using the latter to provide quantitative insight into the adsorption thermodynamics of corrosion inhibitors in acidic solution. Moreover, the validity of the suggestion that $\theta_{\eta \%}$ can be directly related to the fraction of quenched active corrosion sites, and thus provide accurate $\mathrm{K}_{\mathrm{eq}}$ and $\Delta \mathrm{G}_{\text {ads }}^{\circ}$ values, remains unproven, i.e. $\theta_{\eta \%}$ is not proportional to either $\theta_{\mathrm{XPS}}^{\text {Thione }}$ or $\theta_{\mathrm{XPS}}^{\text {Thiol }}$.

A possible concern about the data presented in Figure 2 (c) is the quality of the fits. Visual inspection suggests that even when using equation (5) there is room for improvement. A possible solution would be to use a more complex function to describe the data (e.g. summing more than two Langmuir adsorption isotherms). 
Such an approach, however, cannot be justified based on the current data, and so the significance of any output would be minimal. Interestingly, we have undertaken further fitting of the data with equation (5), but focused only on $\theta_{\mathrm{XPS}}^{\mathrm{Sum}}$, i.e. we relaxed the constraint that we must also fit the $\theta_{\mathrm{XPS}}^{\text {Thione }}$ and $\theta_{\mathrm{XPS}}^{\text {Thiol }}$ profiles well. An improved fit to $\theta_{\mathrm{XPS}}^{\text {Sum }}$ was obtained, but the three fitting parameters were highly correlated, and so the resulting values of $K_{\text {eq }}$ an $\Delta \mathrm{G}_{\text {ads }}^{\circ}$ were very ill-defined.

Having established that determining $K_{\text {eq }}$ and $\Delta G_{\text {ads }}^{\circ}$ from fitting of $\theta_{\eta \%}$ data with the Langmuir adsorption isotherm is not a reliable method for a corrosion inhibitor in acidic solution, the validity of employing more direct estimates of surface coverage (e.g. XPS data) for a similar purpose requires consideration. As indicated in the Introduction, four criteria (listed as (i) - (iv)) need to be sufficiently satisfied for output to be quantitively accurate.

Concerning (i), a maximum adsorbate coverage of a monolayer, it should be possible to directly ascertain this information from the measurements, although demonstrating that it is a single monolayer may not always be straightforward, e.g. a bi-layer may be formed. Turning to (ii), dynamic equilibrium has been achieved, this condition is strictly never realized, as even a well inhibited substrate is continuously corroding. Instead, at best, steady-state is achieved, which may suffice as an approximation to the equilibrium state, although one should demonstrate that this is the case. For example, as employed here, a temporally invariant OCP could be used as evidence.

Focusing on (iii), all adsorption sites are equivalent, the heterogeneity of a corroding substrate (e.g. step edges, grain boundaries, multiple phases) essentially guarantees that this requirement is not fulfilled. Does this invalidate application of the Langmuir isotherm? One could suggest that this heterogeneity can simply be accepted as inevitable, and that the resulting $\mathrm{K}_{\mathrm{eq}}$ and $\Delta \mathrm{G}_{\mathrm{ads}}^{\mathrm{o}}$ are average values. In some scenarios, however, this approximation is almost certainly inappropriate. For example, it has 
been concluded that for the system considered here (i.e. carbon steel in $1 \mathrm{M} \mathrm{HCl}$ inhibited with MBI) the substrate exhibits an iron chloride surface film at lower MBI concentrations, which is not apparent once effective inhibition is achieved $[9,10]$. Adsorption energetics are likely to systematically vary in concert with this change (e.g. $\Delta \mathrm{G}_{\text {ads }}^{\circ}$ for MBI may be lower on the surface terminated by iron chloride), and so obtaining thermodynamic quantities through fitting with a Langmuir isotherm over a range of MBI concentrations is inappropriate.

As regards (iv), an absence of any significant adsorbate-adsorbate interaction, it is well known that organic adsorbates do often interact with each other $[3,14]$. Such adsorbate-adsorbate interactions may be reflected in their adsorption geometry e.g. a more upright orientation at higher surface coverages [14]. Again, such a change may detrimentally interfere with the extraction of $\mathrm{K}_{\mathrm{eq}}$ and $\Delta \mathrm{G}_{\mathrm{ads}}^{\circ}$, using the Langmuir adsorption isotherm.

Given the above, it is hopefully apparent that one must be very careful about quantitative elucidation of corrosion inhibitor adsorption thermodynamics through fitting data with a Langmuir isotherm. Even a more direct estimate of fractional surface coverage than $\theta_{\eta} \%$ may not provide reliable output. One must explicitly demonstrate that the various criteria associated with proper application of the Langmuir isotherm are adequately fulfilled. It should be remembered, as eloquently discussed by Latour [8], that a good fit with the Langmuir isotherm does not indicate that it is an appropriate model for the adsorption process, i.e. correlation does not imply causation. It would perhaps be more appropriate for many acid corrosion inhibitor systems to treat the value obtained for $\mathrm{K}_{\mathrm{eq}}$ as a semi-quantitative descriptor of the isotherm shape rather than as input for determining $\Delta \mathrm{G}_{\text {ads }}^{\circ}$.

Evidently, we have focused solely on the Langmuir isotherm in this paper, and have not commented upon the application of any alternative for describing corrosion 
inhibitor adsorption in acidic solution, e.g. Frumkin or Freundlich adsorption isotherms [6]. It may be argued that using another isotherm model would address some, at least, of the issues raised. An improved fit to the data may result, but again it should be stressed that good agreement does not indicate that an appropriate model has been selected. Indeed, independent of the isotherm employed, one needs to demonstrate that the experimental data are directly related to surface coverage, and that the criteria for employing the selected isotherm model are fulfilled by the system being studied.

Finally, one could contend that the example considered in this study, carbon steel inhibited with $\mathrm{MBI}$ in $1 \mathrm{M} \mathrm{HCl}$, is a singular counter-example, and that more generally we may use $\eta \%$ data to obtain $\mathrm{K}_{\mathrm{eq}}$ and $\Delta \mathrm{G}_{\text {ads }}^{\circ}$. This assertion is worthy of consideration, although it is ultimately flawed. We do not dispute that for some corrosion inhibitor systems $\eta \%$ may be directly proportional to $\theta$, and that the criteria for application of an adsorption isotherm are potentially sufficiently fulfilled. Supporting evidence, however, is required, i.e. one should not simply extract $\mathrm{K}_{\mathrm{eq}}$ and $\Delta \mathrm{G}_{\text {ads }}^{\circ}$ from $\eta \%$ data without explicit validation of the underlying assumptions.

\section{Acknowledgements}

The authors thank Prof. Stuart Lyon for his very constructive comments on the draft manuscript. AkzoNobel and Nouryon supported the work through a collaboration with The University of Manchester. P.M.-G. is grateful to the Fondo Sectorial CONACYT - SENER Hidrocarburos and the Instituto Mexicano de Peteróleo for financial support. 


\section{Data Availability}

The raw data for this study have been published elsewhere $[9,10]$. Experimental data points plotted in Figure 1 and Figure 2 are listed in Supplementary Material. 


\section{References}

1. V.S. Sastri, Corrosion Inhibitors Principles and Applications, Wiley, Chichester, 1998.

2. R. Lindsay, S.B. Lyon, Introduction to Control of Corrosion by Environmental Control, in: R. A. Cottis, M.J. Graham, R. Lindsay, S.B. Lyon, J.A. Richardson, J.D. Scantlebury, F.H. Stott (Eds.), Shreir's Corrosion, vol. 4, Management and Control of Corrosion, Elsevier, Amsterdam, 2010, pp. 2891-2899.

3. A. Kokalj, D. Costa, Molecular Modeling of Corrosion Inhibitors, in: K. Wandelt (Ed.), Encyclopedia of Interfacial Chemistry, Surface Science and Electrochemistry, Elsevier, Amsterdam, 2018, pp 332-345.

4. http://apps.webofknowledge.com (accessed 14 January 2019).

5. W. Al Zoubi, S.G. Mohamed, A.A.S Al-Hamdani, A.P. Mahendradhany, Y.G. Ko, Acyclic and cyclic imines and their metal complexes: recent progress in biomaterials and corrosion applications, RSC Adv. 8 (2018) 23294-23318.

6. Y. Zhu, M.L. Free, R. Woollam, W. Durnie, A review of surfactants as corrosion inhibitors and associated modelling, Prog. Mater. Sci. 90 (2017) 159-223.

7. I. Langmuir, The constitution and fundamental properties of solids and liquids, J. Am. Chem. Soc. 38 (1916) 2221-2295.

8. R.A. Latour, The Langmuir isotherm: A commonly applied but misleading approach for the analysis of protein adsorption behaviour, J. Biomed. Mater. Res. A, 103A (2015) 949-958.

9. P. Morales-Gil, M.S. Walczak, R.A. Cottis, J.M. Romero, R. Lindsay, Corrosion inhibitor binding in an acidic medium: Interaction of 2-mercaptobenzimidazole with carbon-steel in hydrochloric acid, Corros. Sci. 85 (2014) 109-114.

10. P. Morales-Gil, M.S. Walczak, C. Ruiz Camargo, R.A. Cottis, J.M. Romero, R. Lindsay, Corrosion inhibition of carbon-steel with 2-mercaptobenzimidazole in hydrochloric acid, Corros. Sci. 101 (2015) 47-55. 
11. N. Kovačević, I. Milošev, A. Kokalj, The roles of mercapto, benzene, and methyl groups in the corrosion inhibition of imidazoles on copper: II. Inhibitor-copper bonding, Corros. Sci. 98 (2015) 457-477.

12. I. Milošev, N. Kovačević, A. Kokalj, Effect of mercapto and methyl groups on the efficiency of imidazole and benzimidazole-based inhibitors of iron corrosion, Acta Chim. Slov. 63 (2016) 544-559.

13. M.S. Walczak, P. Morales-Gil, T. Belashehr, K. Kousar, P. Arellanes Lozada, R. Lindsay, Determining the Chemical Composition of Corrosion Inhibitor/Metal Interfaces with XPS: Minimizing Post Immersion Oxidation, J. Vis. Exp. 121 (2017) e55163.

14. H. Heinz, C. Pramanik, O. Heinz, Y. Ding, R.K. Mihsra, D. Marchon, R.J. Flatt, I. Estrela-Lopis, J. Llop, S. Moya, R.F. Ziolo, Surf. Sci. Rep. 72 (2017) 1-58. 


\section{Figure Captions}

Figure 1 (a) $\eta \%$ (red markers) and MBI surface coverage from XPS data (blue markers) plotted as a function of MBI concentration for carbon steel after $4 \mathrm{~h}$ immersion in $1 \mathrm{M} \mathrm{HCl}$. Inset shows the thiol and thione

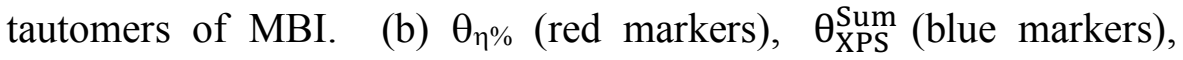
$\theta_{\mathrm{XPS}}^{\text {Thione }}$ (green markers), and $\theta_{\mathrm{XPS}}^{\text {Thiol }}$ (purple markers) plotted as a function of MBI concentration for carbon steel after $4 \mathrm{~h}$ immersion in $1 \mathrm{M} \mathrm{HCl}$. In both (a) and (b), the broken lines are simply guides for the eye.

Figure 2 (a) Best fits (broken lines) with equation (3) to $\theta_{\eta \%}$ (red markers) and $\theta_{\mathrm{XPS}}^{\text {Sum }}$ (blue markers). (b) Values of $\mathrm{K}_{\mathrm{eq}}$ and $\Delta \mathrm{G}_{\mathrm{ads}}^{\circ}$ corresponding to the best fits in (a). (c) Best fits (broken lines) with equation (5) to $\theta_{\text {XPS }}^{\text {Sum }}$ (blue markers), $\theta_{\text {XPS }}^{\text {Thione }}$ (green markers), and $\theta_{\text {XPS }}^{\text {Thiol }}$ (purple markers). (d) Values of $K_{e q}$ and $\Delta G_{\text {ads }}^{o}$ corresponding to the best fits in $(\mathrm{c})$. 

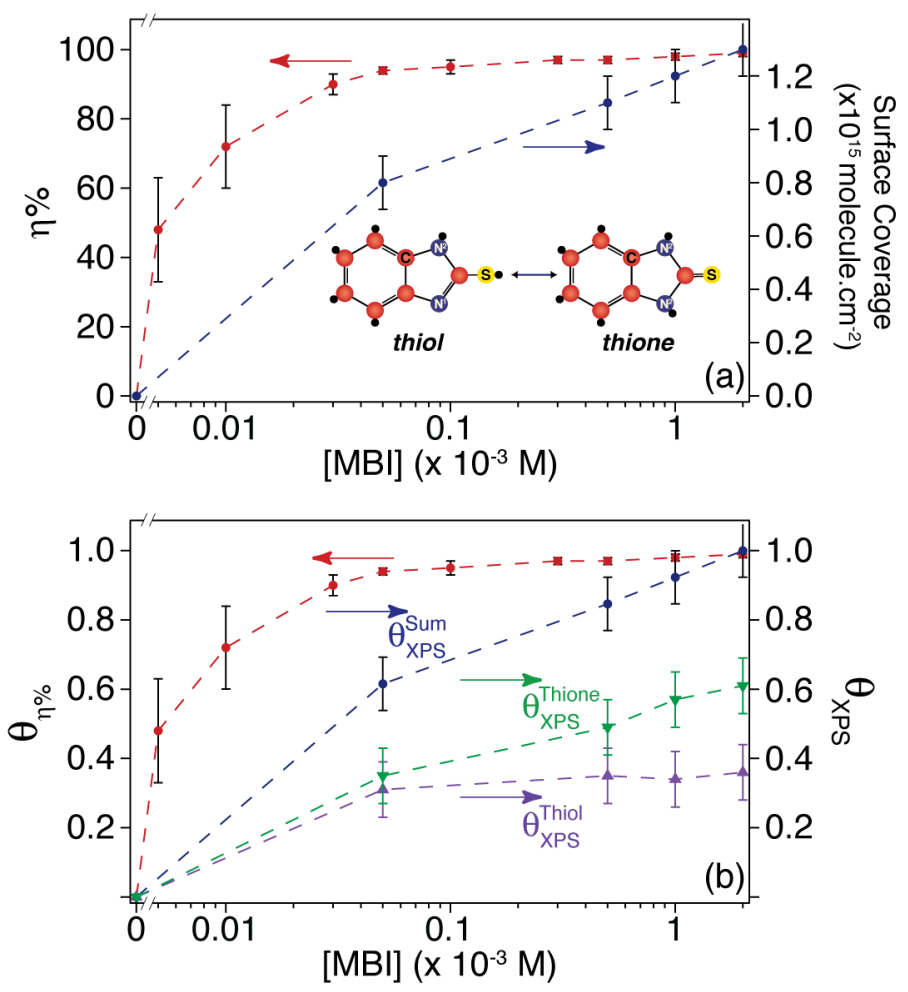

[MBI] $\left(x 10^{-3} \mathrm{M}\right)$

Figure 1 


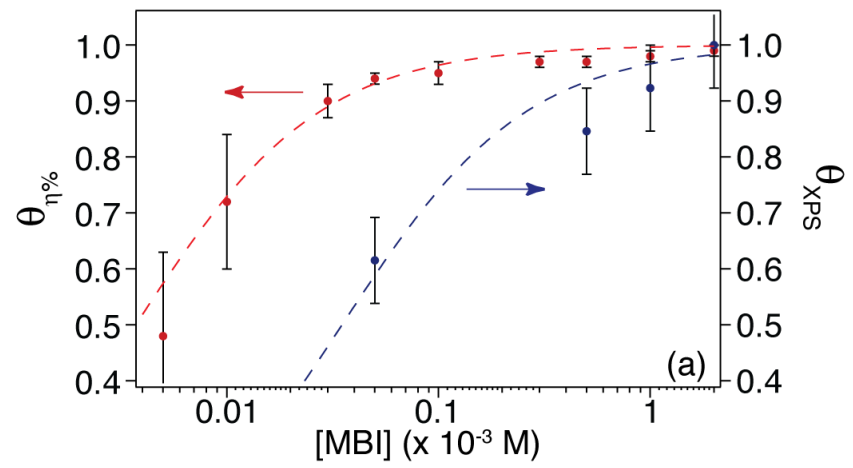

$$
\begin{gathered}
\theta_{\eta \%} \\
\mathrm{~K}_{\mathrm{eq}}=2.7 \times 10^{5} \pm 3 \times 10^{4} \mathrm{M}^{-1} \\
\Delta \mathrm{G}^{\circ}{ }_{\text {ads }}=-31.0 \pm 0.3 \mathrm{kJmol}^{-1} \\
\theta_{\text {XPs }}^{\text {Sum }} \\
\mathrm{K}_{\mathrm{eq}}=2.9 \times 10^{4} \pm 9 \times 10^{3} \mathrm{M}^{-1} \\
\Delta \mathrm{G}^{\circ}{ }_{\text {ads }}=-25.5 \pm 0.8 \mathrm{kJmol}^{-1}
\end{gathered}
$$
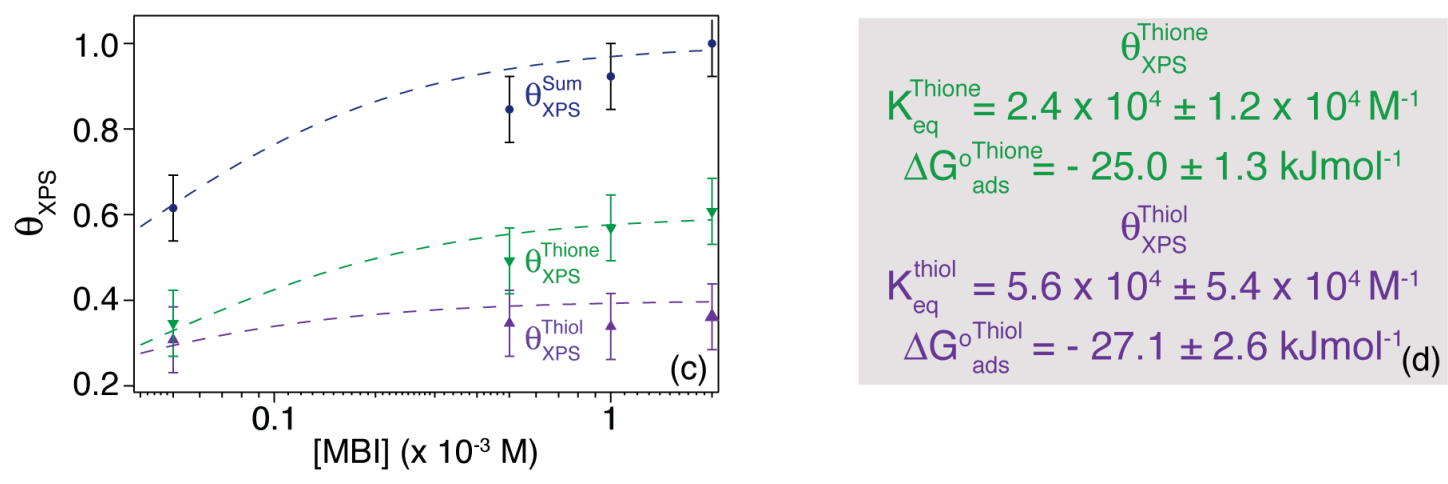

Figure 2 